\title{
PLANNED NEW COOLER/STORAGE RING COMPLEX FOR RADIOACTIVE BEAMS AND ANTIPROTONS AT GSI
}

\author{
F. Nolden, K. Beckert, P. Beller, O. Boine-Frankenheim, A. Dolinskii, \\ B. Franzke, M. Steck, H. Weick, GSI, Darmstadt, Germany
}

\begin{abstract}
Radioactive ions (RIs) and antiprotons (pbars) play a major role in the new planned GSI facility. After shooting $1 \mathrm{GeV} / \mathrm{u}$ pulses of up to $1 * 10^{12}$ primary ions with a length of $50 \mathrm{~ns}$ on a production target, secondary RI beams are prepared in a superconducting fragment separator. Pbars originate from a primary $30 \mathrm{GeV}$ proton beam of $1.5 * 10^{13}$ particles. A dedicated collector ring (CR) serves for precooling of RI's at $740 \mathrm{MeV} / \mathrm{u}$ and of pbars at $3 \mathrm{GeV}$. Active debunching and stochastic cooling provide beams of sufficient quality. In order to fulfill the different requirements of stochastic cooling of the two types of beam, a novel split ring lattice design is applied. Isochronous RI mass measurements are possible in the CR, too. A second ring, the New Experimental Storage Ring, serves for beam accumulation and, in the case of RI beams, internal experiments. Optimum conditions for RI experiments are reached by electron cooling. An internal target as well as an electron nucleus scattering facility open up new frontiers for the investigation of nuclear properties far from stability. The pbar beams are accumulated by stochastic cooling. The paper discusses beam physics aspects of the new storage rings.
\end{abstract}

\section{RINGS FOR RADIOACTIVE IONS AND ANTIPROTONS}

The heavy ion research institute GSI of Darmstadt, Germany is planning to upgrade its present facility [1]. Among the main thrusts of research are nuclear physics studies with unstable nuclei and physics with antiproton beams at high energy (up to $15 \mathrm{GeV}$ ). Both are secondary beams which are produced by shooting intense beams with a short pulse duration of $50 \mathrm{~ns}$ on dedicated production targets. The details of the primary beam production are published elsewhere [2]. Here we present some results of a study which is still going on, concerning the dedicated rings which are planned to cool and accmulate these secondaries.

For the purpose of efficient preparation of high-intensity secondary beams, the two-storage ring concept is adopted, which was effective at the CERN AC/AA-complex and is applied at the FNAL debuncher and accumulator, as well. A special feature of the GSI concept is that the rings shall be able to cool both antiprotons and radioactive ions beams. Our collector ring is called CR, the accumulator ring is the NESR. Stochastic cooling will be used exclusively in the $\mathrm{CR}$ and for antiproton accumulation in the NESR. RI accumulation in the NESR will use electron cooling. The maximum magnetic rigidity of both rings is $13 \mathrm{Tm}$.

Antiprotons which emerge at an energy of $3 \mathrm{GeV}$ are accepted in the CR. After accumulation in the NESR, they will be used for internal target experiments in a dedicated high energy storage ring [1].

The RI specific energy is $740 \mathrm{MeV} / \mathrm{u}$. A new superconducting fragment separator allows to select single secondary nuclear species for injection into the CR. The NESR will be used for both accumulation and internal experiments with RIs.

The rings will be used continuously for either species on a time scale of at least several weeks. In order to use most of the hardware for both species,

- the polarity of ring magnets can be inverted,

- the injection and extraction hardware, as well as the rf systems are usable for both RIs and pbars,

- the optical settings are flexible enough to be fitted to specific requirements, as beam cooling or internal experiments.

\section{COLLECTOR RING}

The collector ring is a large acceptance ring which has three different optical settings in order

- to precool RIs at $740 \mathrm{MeV} / \mathrm{u}$,

- to precool antiprotons at $3 \mathrm{GeV}$,

- to perform mass measurements of short-lived nuclei by measuring their revolution frequency. The ring is tuned to transition in that mode (isochronous setting), in order to get rid of the frequency spread due to different momenta. This method has successfully been applied at GSI's experimental storage ring ESR [3].

The CR circumference is $188 \mathrm{~m}$. It accepts the secondary beams as they emerge from their production target in a single bunch of $50 \mathrm{~ns}$ length. A full aperture kicker is used to fill the whole horizontal acceptance of the ring. Bunch rotation and active debunching reduce the momentum width to a value which is acceptable for the stochastic cooling system. The secondaries are then cooled by stochastic cooling. Finally the beam is rebunched and transferred to the next ring, the NESR. Fig. 1 shows the general layout of the ring. The beam circulates clockwise.

Table 1 shows the beam parameters after injection, after active debunching and after stochastic cooling. The RI final 


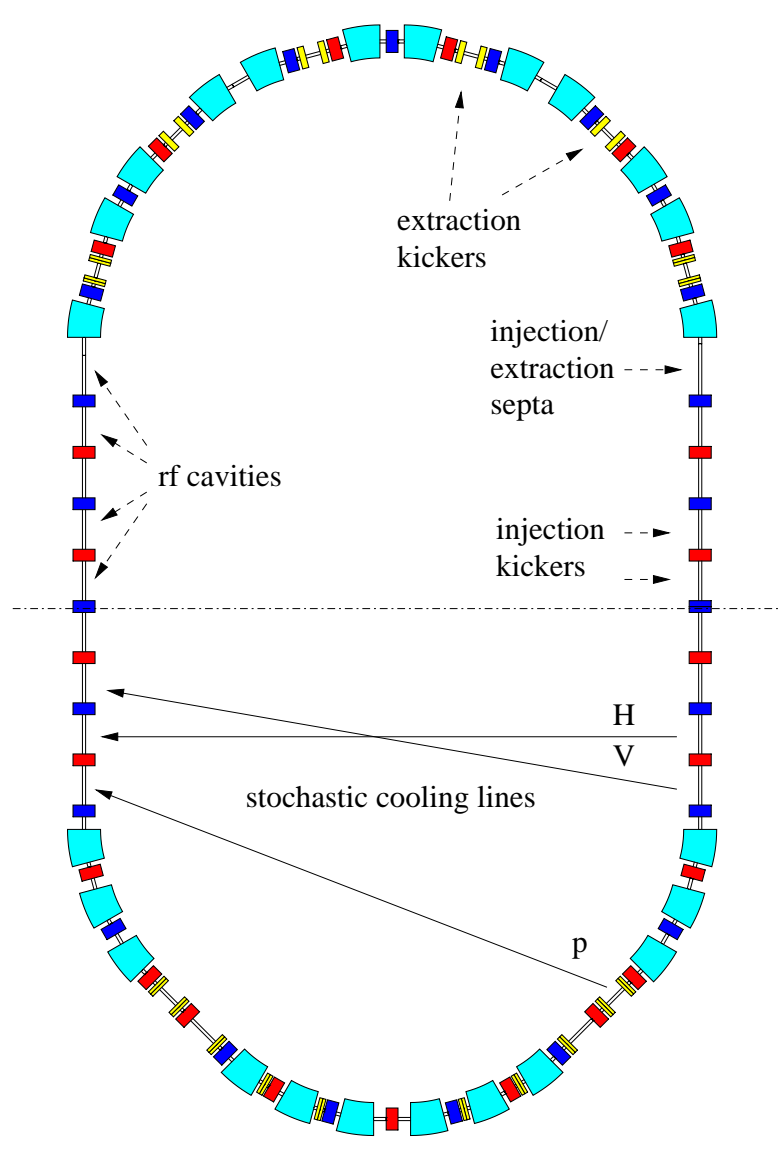

Figure 1: Layout of CR Ring

phase space is sufficient for fast magnetized electron cooling in the NESR. The antiproton parameters are the same as in the CERN AC [5].

\begin{tabular}{|c|c|c|c|c|}
\hline & \multicolumn{2}{|c|}{$\delta p / p$} & \multicolumn{2}{c|}{$\epsilon_{x y}[\pi \mathrm{mm}$ mrad $]$} \\
& RIs & pbars & RIs & pbars \\
\hline injected & $\pm 2.5 \%$ & $\pm 3.0 \%$ & 50 & 240 \\
debunched & $\pm 0.5 \%$ & $\pm 0.7 \%$ & 50 & 240 \\
cooled & $\pm 0.05 \%$ & $\pm 0.1 \%$ & 0.5 & 5 \\
\hline
\end{tabular}

Table 1: Parameters of beam cooling in the $\mathrm{CR}$

A general problem of fast stochastic cooling is the reduction of undesired mixing between pick-up and kicker. Due to this effect, the cooling force at a certain frequency $\Omega$ is reduced by a factor $\cos \Omega \tau_{\text {bad }}$, because the signal arrival at the kicker and the particle arrival time differ by a delay $\tau_{\text {bad }}$, which is given by

$$
\tau_{\text {bad }}=-\eta_{p-k} T_{p-k} \frac{\delta p}{p_{0}}
$$

$T_{p-k}$ is the nominal time of flight between pick-up and kicker.The local slip factor is

$$
\eta_{p-k}=\gamma^{-2}-\alpha_{p-k}
$$

with the local momentum compaction

$$
\alpha_{p-k}=\frac{1}{s_{k}-s_{p}} \int_{s_{p}}^{s_{k}} \frac{D(s)}{\rho(s)} d s
$$

Here, $s_{k}-s_{p}$ denotes the path length between pick-up and kicker, $D(s)$ is the dispersion function and $\rho(s)$ is the orbit curvature. In order to cool efficiently, $\Omega \tau_{\text {bad }}$ must be considerably smaller than $\pi / 2$.

On the other hand it is necessary to reduce the diffusion due to Schottky noise. The diffusion does not only broaden the equilibrium momentum distribution of longitudinal stochastic cooling; it also enlarges the achievable transverse emittances. This heating is proportional to the Schottky power density at the revolution harmonics where the cooling system is working. It is inversely proportional to the total frequency slip $\eta_{\text {tot }}$, as long as the Schottky bands do not overlap. The revolution frequency slip factor $\eta_{\text {tot }}$ is calculated in analogy to the local $\eta_{p-k}$, but the momentum compaction factor must be taken over a full revolution.

From these general considerations arises the principle of the split ring design, which was pioneered with the SuperLEAR proposal in the early 90's at CERN [4]. The main consideration is to split the ring in such a way that the optics is locally close to transition $\left(\eta_{p-k} \approx 0\right)$ between pickup and kicker, and that it is far away from it (below or above transition) in the rest of the ring.

The CR lattice consists of two opposite arcs with two long straight sections in between. Because of the different Lorentz factors for RIs $(\gamma=1.7)$ and antiprotons $(\gamma=4)$, the reduction of $\eta_{p-k}$ leads to very different values of the average dispersion between pick-up and kicker. Only the lower arc in fig. 1 is varied in its optical setting, when RI beams are replaced by antiprotons or vice versa. The upper arc has a constant optical setting, which has the advantage that injection and extraction elements in this arc can be used with the same setting for both antiprotons and RIs. All stochastic cooling kickers and all rf cavities are installed in straight sections with zero dispersion. Table 2 gives a quantitative view of the features discussed above. The symbol $\mathrm{p}$ in table 2 and fig. 1 refer to the longitudinal (Palmer) cooling line, $\mathrm{H} / \mathrm{V}$ to the horizontal and vertical cooling lines, and full to the parameters for one revolution.

\begin{tabular}{|c|c|c|c|c|c|c|}
\hline & \multicolumn{2}{|c|}{$\alpha_{p-k}$} & \multicolumn{2}{c|}{$\eta_{p-k}$} & \multicolumn{2}{c|}{$T_{p-k}[\mathrm{~ns}]$} \\
\hline & RIs & pbars & RIs & pbars & RIs & pbars \\
\hline $\mathrm{p}$ & 0.177 & 0.062 & 0.134 & -0.005 & 196 & 168 \\
\hline $\mathrm{H} / \mathrm{V}$ & 0.138 & 0.061 & 0.138 & -0.004 & 283 & 242 \\
\hline full & 0.066 & 0.035 & 0.245 & 0.021 & 726 & 620 \\
\hline
\end{tabular}

Table 2: Mixing parameters for CR stochastic cooling

The present stochastic cooling concept for the RI beams uses a band of 0.9-1.7 GHz which should be sufficient to reach e-folding cooling times of less than $100 \mathrm{~ms}$ for $10^{7}$ 
injected RIs in a high charge state $q>25$. The high charge state guarantees an excellent signal to noise ratio. Fast stochastic cooling of heavy ions has been achieved at GSI [6]. The stochastic cooling scenario for RIs results from the residual undesired mixing. Palmer cooling will be applied for longitudinal cooling until a momentum spread of $\pm 3 \times 10^{-3}$ remains. Then the transverse cooling systems will be turned on. It is envisaged to change to longitudinal notch filter cooling in the very last stage, when a momentum spread of $\pm 1 \times 10^{-3}$ has been reached.

The parameters for the antiprotons are similar to those of the CERN AC, so that a similar cooling concept can be applied. Cooling will begin in the $0.9-1.7 \mathrm{GHz}$ band. The upper working frequency will then be moved to $4 \mathrm{GHz}$ as soon as the undesired mixing allows it. Longitudinal cooling will use the notch filter method from the beginning.

If the CR is operated on transition, i.e. $\gamma \approx \gamma_{T}=1.7$, the dispersion function has also to be raised in the upper arc. This reduces the transverse acceptances to $50 \pi \mathrm{mm}$ mrad at a momentum spread of $\pm 3 \times 10^{-3}$.

\section{NEW EXPERIMENTAL STORAGE RING}

The NESR is a versatile storage ring which will be used both for internal experiments with RIs and for the accumulation of antiprotons, as well. Its circumference is $208 \mathrm{~m}$.

An important goal of the internal experiments is the determination of electric charge distributions of unstable nuclei from elastic electron-nucleus scattering. There will be a dedicated collision experiment inside the NESR with an electron beam circulating in a small electron storage ring $(100 \mathrm{MeV}-500 \mathrm{MeV})$ with a circumference of roughly $30 \mathrm{~m}$. The electron ring as well as the optical design of the collision zone is being worked out in collaboration with the Budker Institute at Novosibirsk in Russia. A second experiment will involve scattering off an internal gas jet. Atomic physics experiments will be possible with an additional free electron target. Fig. 2 shows the present layout of the ring.

Electron cooling will prepare and preserve RI beams of high phase space density. The NESR electron cooler will operate at energies of up to $410 \mathrm{keV}$. According to the experience at GSI's ESR storage ring, final emittances of $0.1 \pi \mathrm{mm}$ mrad at a momentum spread of less than $10^{-4}$ will be possible, unless the ion beam density exceeds some $10^{6} \mathrm{ions} / \mathrm{m}$. The necessary electron beam density of $5 \times 10^{7} \mathrm{~cm}^{-3}$ will be achieved at an electron current of $1 \mathrm{~A}$, using an electron gun with optimized beam diameter. The guiding field is $0.2 \mathrm{~T}$, and the field parallelity in the cooling section must be better than $5 \times 10^{-5}$. The construction of the electron cooler will require the extrapolation of the experience made with the electron cooler at GSI's experimental storage ring ESR [7].

During antiproton experiments the NESR will serve as an antiproton accumulator. The antiprotons will be $\mathrm{rf}$ stacked, followed by stochastic cooling of the stack tail. Stack core stochastic cooling will serve to move the stack

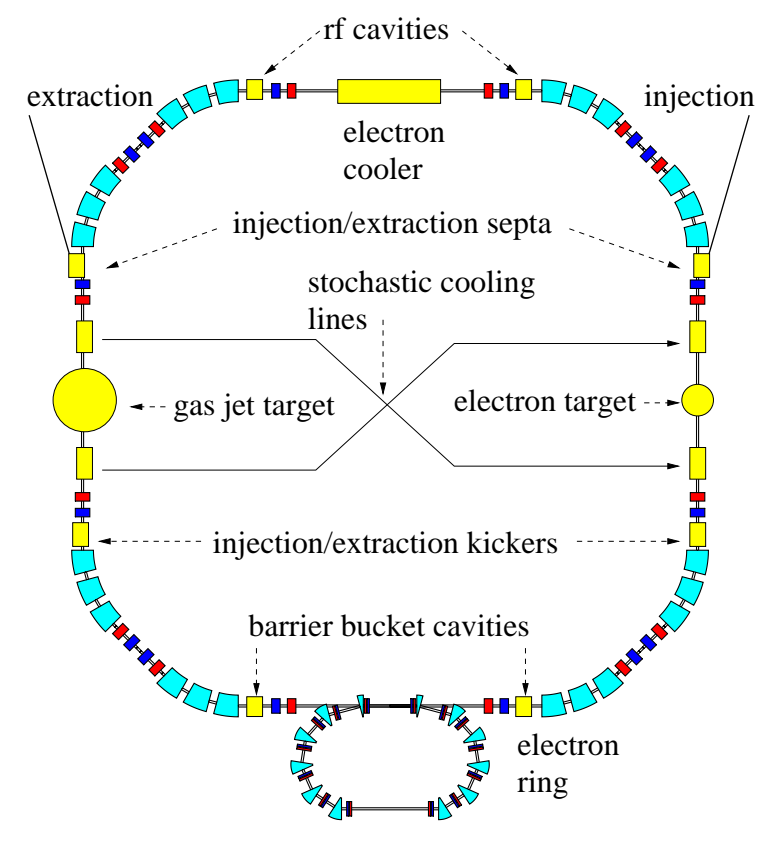

Figure 2: Layout of NESR Ring

tail into the stack, thereby accumulating up to $10^{12}$ antiprotons over a few hours. This technique is well-known and has been applied at CERN and at FNAL. At present, a working bandwidth of up to $8 \mathrm{GHz}$ is under consideration.

\section{FUTURE DEVELOPMENTS}

The design of the cooler rings is still in a preliminary phase. Major research projects in connection with beam cooling are

- the design of bunch rotation cavities for the CR,

- the design of stochastic cooling electrodes for CR and NESR,

- simulation calculations of the cooling process based on the Fokker-Planck approach,

- and the development of the NESR eectron cooler.

These developments will be part of a detailed technical proposal presently under preparation.

\section{REFERENCES}

[1] W. Henning, this conference

[2] P. Spiller et al., this conference

[3] M. Hausmann et al., Nucl. Inst. Meth. A446 (2000), 569

[4] R. Giannini, P. Lefèvre, D. Möhl, Nucl. Phys. A558 (1993) $519 \mathrm{c}-536 \mathrm{c}$

[5] H. Koziol, S. Maury, internal report CERN/PS 95-15 (AR/BD)

[6] F. Nolden et al., Proc. EPAC 2000, 1262-1264

[7] M. Steck et al., Proc. EPAC 1994, 1197 